\title{
Mit kaltem Wasser gegen Alterungsprozesse - „Anti-Aging“ mit Hydrotherapie nach Kneipp
}

\section{Autor: Peter W. Gündling}

\section{ZUSAMMENFASSUNG}

Hydrotherapeutische Verfahren erscheinen vielen umständlich, aufwendig und altmodisch. Doch sie sind alles andere als das. Gerade in der heutigen, chemie- und chirurgiegläubigen Zeit, stellen sie ein wertvolles Instrument im naturheilkundlichen Repertoire dar. Als typische Regulationsverfahren wirken sie ausgleichend auf die Aktivität des vegetativen Nervensystems und tragen so zur Verbesserung des Stoffwechsels und der Durchblutung bei. Zudem aktivieren sie das Immunsystem und die zellulären Reparaturmechanismen und regen die Regulation des Hormonhaushaltes an. Gleichzeitig unterstützen sie - mit relativ geringem Aufwand - die Eigenverantwortung und Selbsthilfefähigkeit der Betroffenen.

Schlüsselwörter

Hydrotherapie, Wasserheilkunde, Regulationsverfahren, Bäder, Teilbäder, Kneipp‘sche Güsse, Sauna

\section{ABSTRACT}

Many people are of the opinion, that hydrotherapeutic procedures are cumbersome, complex and old-fashioned. But they are far from that. Especially today, where people believe in chemistry and surgery, these procedures are a valuable instrument in the naturopathic repertoire. As typical regulatory procedures they have a balancing effect on the vegetative nervous system, thereby contributing to the improvement of the metabolism and the blood flow. Moreover, they activate the immune system and cellular repair mechanisms and stimulate the regulation of the hormonal balance. At the same time, they support - with relatively little effort - the personal responsibility as well as ability of the people affected to help themselves.

Keywords

Hydrotherapy, regulatory procedures, baths, partial baths, Kneipp affusions, sauna

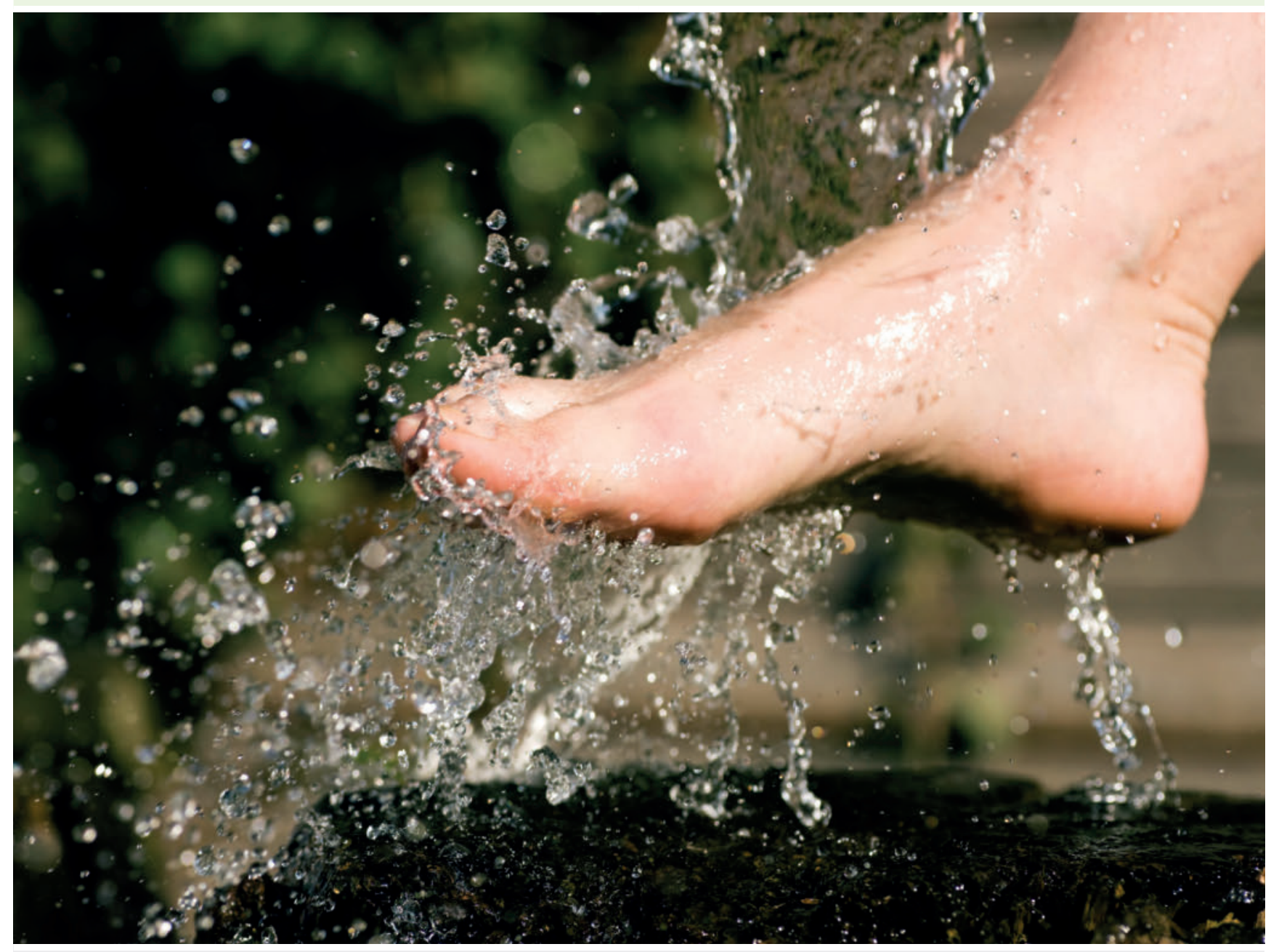

Abb. 1 @ Kzenon/Adobe Stock 
„Wasser thut's freilich“, betitelte der Pionier der deutschen Hydrotherapie Theodor Hahn bereits 1776 sein Buch [14]. Das Buch, das Sebastian Kneipp etwa 50 Jahre später half, sich selbst von seiner damals als unheilbar geltenden Lungentuberkulose zu heilen. Doch hydrotherapeutische Anwendungen regen nicht nur die Selbstheilungskräfte kranker Personen an, sie wirken auch in besonderem Maße präventiv. Durch ihre Reizwirkung und die darauf folgenden Regulationen unterstützen und stimulieren sie auch die Regenerationsmechanismen der physiologischen Alterungsprozesse. Damit stellen sie ein wertvolles Instrument dar, Alterserscheinungen, wie sie sich durch Kreislauf- und Durchblutungsstörungen, Gelenkbeschwerden, nachlassende Gedächtnis- und Sinnesfunktionen sowie Leistungseinbußen und Erschöpfungszustände bemerkbar machen, spürbar entgegenzuwirken.

Bereits Mitte des 19. Jahrhunderts erkannte Sebastian Kneipp, dass viele Krankheiten Folgen des „modernen Lebensstils“ waren. Gleichzeitig verstand er es, die Wasserheilkunde in ein System mit einfacher, gesunder Ernährung, regelmäßiger Bewegung, seelischem Ausgleich (Ordnungstherapie) und Behandlung mit Heilkräutern einzubinden [15][16]. Ein System, dass wir heute „klassische Naturheilverfahren“ nennen.

Dabei ist die Hydrotherapie ein regulationsmedizinisches Verfahren par excellence. Ihr Wirkungsfeld ist das vegetative Nervensystem. Richtig angewandt verbessert sie Mikround Makrozirkulation, regt den Zellstoffwechsel an, verbessert den Gas- und Nährstoffaustausch, moduliert das Immunsystem und schafft einen Ausgleich zwischen Sympathikus und Parasympathikus [5].

Ein Patientenbeispiel: Vor einigen Jahren kam ein evangelischer Theologe in meine Praxis und bat mich - in meiner Funktion als Badearzt - um die Verordnung von Kuranwendungen. Anamnestisch berichtete mir der damals 78-jährige Mann, dass er Anfang der 1940er-Jahre in den Krieg eingezogen werden sollte, aber aufgrund seines schon zu dieser Zeit schwachen Herzens ausgemustert wurde. Als Kind hatte er eine schwere Scharlachinfektion durchgemacht und als Folge wohl eine Endokarditis erlitten. Seitdem war er körperlich kaum belastbar. Um dennoch sein angestrebtes Studium und seinen zukünftigen Beruf bewältigen zu können, begann er, regelmäßige zügige Spaziergänge und - nach der Lektüre von Kneipp auch regelmäßige Wasseranwendungen zu machen. Seit seinem 50. Lebensjahr absolvierte er zudem alle paar Jahre eine Kneippkur. Das habe ihm geholfen, seinen Beruf bis ins hohe Alter auszuüben und halte ihn fit und gesund. Deswegen sei er nun auch in unser Kneippheilbad gekommen. Auf die Frage nach Einnahme von Medikamenten sagte er, dass er das von den Internisten verordnete Zeug nicht nehme. Er wolle sich ja nicht vergiften. Er nehme lediglich $2 \times$ pro Tag Weißdorn, das tue ihm gut.
Bei der körperlichen Untersuchung fiel mir ein 4/6 Systolikum auf, das über allen Ostien auskultierbar war. Sein Blutdruck lag bei $125 / 80 \mathrm{mmHg}$, der BMI bei $23 \mathrm{~kg} / \mathrm{m}^{2}$ und der Puls bei regelmäßig 64/min. Er wirkte nicht nur geistig frisch, er war auch sehr beweglich und zeigte keinerlei Schwäche- oder Insuffizienzzeichen. Hätte ich sein Geburtsdatum nicht auf der Karteikarte gelesen, hätte ich ihn mindestens 10 Jahre jünger geschätzt.

Ich verordnete ihm die ortsüblichen hydrotherapeutischen Anwendungen mit Güssen, Teilbädern, kalten Abwaschungen, Wickeln und Lehmpackungen und bestellte ihn in der Folgewoche zur Kontrolluntersuchung ein. Bei der Wiedervorstellung berichtete er mir, dass er alle Anwendungen gut vertragen habe und in den letzten Tagen schon dreimal hoch zur Kreuzkapelle (einem Aussichtspunkt, der etwa 300 Höhenmeter oberhalb unserer Kurstadt liegt) gelaufen sei. Bei der Abschlussuntersuchung nach drei Wochen wiederholte und bekräftigte er diese Aussagen. Da es ihm bei uns gut gefallen hatte, kam er in den Folgejahren im Abstand von 2-3 Jahren noch einige Male. Als er im Alter von 92 Jahren - immer noch recht fit und rüstig - schließlich das letzte Mal bei uns zur Kur war, verabschiedete er sich mit den Worten: „Meine Kinder meinen, ich wäre jetzt zu alt, um zu vereisen. Sie machen sich Sorgen um mich und ich will sie nicht unnötig beunruhigen.“

Obwohl ich mich an diesen Patienten, auch wegen seines netten Wesens, sehr gut und gerne erinnere, ist er kein Einzelfall. Patienten, die regelmäßig Wasseranwendungen machen, sich genügend bewegen und auch sonst einigermaßen gesund leben, sind durchwegs fitter, haben eine straffere Haut, eine bessere Gesichtsfarbe und Durchblutung und fühlen sich wohler. Machen sie zudem noch regelmäßig entsprechende Kuren, verstärken sich diese Effekte.

\section{Was ist das Besondere an}

\section{Wasseranwendungen - wie wirkt die Hydrotherapie?}

Kaltes wie auch warmes Wasser stimuliert die äußeren Thermorezeptoren, die diese Information über afferente (Aס-)Nervenfasern an das zentrale Thermoregulationszentrum im Hypothalamus weiterleiten. Über motorische und vegetative Fasern kommt es dann je nach Reizstärke zur Dilatation oder (kurzzeitiger) Konstriktion der Gefäße und als Folge zur Anregung der Durchblutung und Wärmebildung der Haut. Über Reflexbahnen erfolgt dann eine Beeinflussung der Skelettmuskulatur und der inneren Organe. Zudem werden immunologische und hormonellhumorale Reaktionen insbesondere des Hypophysen-Nebennierenrinden-Systems angeregt [4][11].

Werden die Reize regelmäßig appliziert, erfolgt ein Anpassungseffekt, bei dem es durch die Downregulation von Adrenalin zu einer dauerhaften Minderung des Sympathi- 
kotonus und einer Regeneration des Regelmechanismus kommt. Durch das regelmäßige Gefäßtraining wird zudem die Vasomotion gefördert und die Endothelfunktion regeneriert [7][12]. Über diese Mechanismen wirkt die Hydrotherapie sowohl durchblutungsverbessernd als auch stoffwechselanregend und zellregenerierend.

\section{Hydrotherapeutische Anwendungen zur Vermeidung vorzeitiger Alterung}

Da die allgemeinen Regeln zur Durchführung von Wasseranwendungen in letzter Zeit auch an dieser Stelle mehrfach beschrieben wurden [3][9][11][12], sollen diese hier nur kurz dargestellt werden: Wärme ist Heilenergie! Deshalb sollte jeglicher Kaltreiz nur auf warmer Haut erfolgen und danach auf Wiedererwärmung - aktiv oder passiv geachtet werden. Der wichtigste Parameter einer richtig dosierten Wasseranwendung ist das Wohlbefinden des Behandelten. Tut eine Anwendung nicht gut, wurde sie nicht korrekt gewählt, ausgeführt oder dosiert.

Individuelle, situationsgerechte Dosierung heißt, den Unterschied zwischen Wasser- und Körpertemperatur, die Größe der behandelten Körperoberfläche, die Dauer der Anwendung und aufgrund des zirkadianen Rhythmus der Körpertemperatur auch die gewählte Tageszeit zu beachten. Kalte Anwendungen wirken morgens intensiver, warme abends. Nicht zuletzt ist auch die Konstitution des zu Behandelnden zu beachten. Während schlanke, schmalwüchsige Menschen (sog. Astheniker) stärker wärmebedürftig sind, vertragen vollblütige, eher untersetzte Menschen (sog. Pykniker) kräftige und größere Kaltanwendungen. Während diese beiden Typen meist richtig beurteilt werden, werden körperlich starke Menschen (sog. Athletiker) häufig falsch eingeschätzt. Sie sind meist kälte- und wärmesensibler als erwartet und sollten demnach milde Reize erhalten.

\section{Trockenbürsten}

Strenggenommen gehört das Trockenbürsten, d. h. die Massage des Körpers mit einer Bürste oder einem rauen Tuch, nicht zu den Wasseranwendungen. Dennoch wird es wegen der Art der Durchführung und der ähnlichen Wirkungen dazu gezählt. Es wirkt lokal auf die Haut und das Bindegewebe und allgemein und reflektorisch auf die inneren Organe durchblutungsfördernd, blutdruckregulierend, herzentlastend, vegetativ stabilisierend und psychisch aktivierend. Darüber hinaus regt es den Zellstoffwechsel an und wirkt hauterneuernd, -regenerierend und -tonisierend.

Als klassischer Anti-Aging-Effekt zeigt sich eine straffere, geschmeidigere und besser durchblutete Haut. Besonders zu empfehlen ist Trockenbürsten morgens sofort nach dem Aufstehen, am besten in frischer Luft bzw. vor dem offenen Fenster, da es aktivierend wirkt. Benötigt werden dazu eine Bürste aus Naturfaser oder Sisal mit Schlaufe oder Handgriff und langem abnehmbaren Stiel oder ein raues Handtuch sowie etwa fünf Minuten Zeit.
Kontraindiziert ist es bei entzündlichen Hautkrankheiten, Akne, Hautverletzungen, Thrombophlebitis, ausgeprägter Varikosis, Ulcus cruris (diese Gebiete aussparen!), überstarker Körperbehaarung, Polyneuropathie und nervöser Übererregbarkeit.

Begonnen wird die Behandlung am rechten Fußrücken, dann wird sie kreisförmig über die Fußsohle, den rechten Unterschenkel zum rechten Oberschenkel - erst Außen-, dann Innenseite - durchgeführt. Dabei sollte der Druck jeweils zum Herzen hin verstärkt werden. Danach folgen das linke Bein in gleicher Weise und schließlich das Gesäß. Anschließend folgen der Oberkörper, beginnend am rechten Handrücken und Arm, erst Außen-, dann Innenseite (in Längsrichtung), dann der linke Handrücken und Arm, erst Außen- dann Innenseite, dann die Brust zum Brustbein hin und der Nacken zur Schulter hin, schließlich der obere und der untere Rücken. Zum Schluss folgt das Gesicht mit einer besonders weichen Bürste. Die Behandlung wird jeweils solange durchgeführt, bis eine leichte Rötung der Haut eintritt [1][5].

\section{Wassertreten - erfrischt am Tage, beruhigt am Abend}

Die wohl bekannteste Kneipp'sche Anwendung ist das Wassertreten. Es kann nahezu überall durchgeführt werden, ob in einem speziellen Wassertretbecken, einem seichten Bachlauf oder einer Badewanne. Besonders bietet es sich in Verbindung mit einem (Abend-)Spaziergang, (Nordic-)Walking oder anderer körperlicher Aktivität an. Es tonisiert das Gewebe, fördert den venösen Rückstrom, wirkt reaktiv hyperämisierend und erwärmend. Bei regelmäßiger Anwendung wirkt es zudem vegetativ ausgleichend, beugt Infekten vor, regt den Stoffwechsel an und wirkt beruhigend und schlaffördernd am Abend.

Im Rahmen von Anti-Aging ist Wassertreten besonders bei Einschlafstörungen zur Verbesserung der arteriellen und venösen Durchblutung sowie bei funktionellen HerzKreislauf-Beschwerden angezeigt. Kontraindiziert ist es bei Menstruation und Unterleibsinfektionen der Frau, Blasenund Nierenkrankheiten, arteriellen Durchblutungsstörungen schweren Grades (AVK Grad IIb-IV) sowie Kältegefühl und kalten Füßen.

Die Durchführung ist einfach: Hosenbeine hochkrempeln oder Rock hochhalten und im „Storchengang“, also bei jedem Schritt ein Bein ganz aus dem Wasser hebend, durch das Wasser steigen. Je nach Wassertemperatur reichen bereits 1-2 Minuten. Danach das Wasser abstreifen, ggf. Zwischenzehenräume abtrocknen und sofort Strümpfe und Schuhe anziehen. Wiedererwärmung durch Laufen oder abends im Bett [9][12].

\section{Kalte Waschung}

Da erholsamer Schlaf eine wesentliche Voraussetzung für die (nächtliche) Regeneration der Gewebe ist, soll ergän- 
zend zur Empfehlung des abendlichen Wassertretens hier noch eine Anwendung angeführt werden, mit der Sebastian Kneipp sogar den damaligen Papst Leo XIII. von seinen Schlafstörungen befreit haben soll. Das Stresshormon Kortisol ist nicht nur ein Gegenspieler des Melatonins, sondern auch des Wachstumshormons (Somatotropes Hormon, STH). Das STH wiederum ist wesentlich für die Zellregeneration. Somit bewirken eine Umstimmung und Harmonisierung des vegetativen Nervensystems nicht nur einen verbesserten Schlaf, sondern auch eine bessere Regeneration. Genau diese vegetative Umstimmung plus eine reaktive Umverteilung des Blutes vom Kopf in die Peripherie - und damit eine Beruhigung des Geistes - ist das Ziel einer kalten Waschung. Mit Blick auf den Anti-Aging-Effekt wirkt der sanfte Wasserreiz darüber hinaus stimulierend auf den Hautstoffwechsel und verbessert die Entgiftung über die Haut.

Während Ganz- oder Oberkörperwaschungen meist frühmorgens vor dem Aufstehen im Bett durchgeführt werden, dient die entspannende Unterkörperwaschung besonders als Einschlafhilfe. Dazu wird vor dem Einschlafen mit einem Waschhandschuh oder Tuch aus Leinen ein dünner Wasserfilm auf die Haut aufgetragen. Um sicherzustellen, dass der Körper auch warm genug ist und nach der Anwendung schnell wieder warm wird, sollte man sich vor der Waschung zunächst so lange ins Bett legen, bis sich Bett und Körper wohlig warm anfühlen. Um die Waschung durchzuführen, steht der Patient kurz auf, taucht das Leinentuch in kaltes Wasser, wringt es aus und beginnt am rechten Fußrücken. Dann das Tuch über den Außenknöchel, die Außen- und Rückseite des Unter- und Oberschenkels bis zum Gesäß und über den Beckenkamm bis zum Bauch führen. Zwischendurch das Tuch wieder in das Wasser eintauchen, kurz auswringen und es über die Haut auf der Beininnen- und -vorderseite hinunterführen, bis das gesamte rechte Bein von einem Wasserfilm umhüllt ist. In der gleichen Weise wird die linke Unterkörperhälfte gewaschen: Unten außen beginnen und unten innen be- enden. Danach nicht abtrocknen, Schlafkleidung anziehen, schnell ins warme Bett und gut zudecken.

Wer Probleme damit hat, nach der kalten Waschung wieder warm zu werden, kann dem kalten Wasser einen Schuss Essig oder einen Löffel Meersalz zusetzen. Das reizt leicht die Haut und führt zu einer intensiveren Erwärmung.

\section{Kalter Gesichtsguss - der „Schönheitsguss“}

Güsse sind das Herzstück der Kneipp'schen Hydrotherapie und werden idealerweise mit einem Schlauch von 1,5 m Länge und 3/4 Zoll (20 mm) Durchmesser oder einem Gießhandstück, das anstelle des Brausekopfes auf den Duschschlauch geschraubt wird, durchgeführt. Sollte beides nicht zur Verfügung stehen, funktioniert ersatzweise auch eine weichgestellte Handbrause. Zur Selbstanwendung empfehlen sich sogenannte Flachgüsse, die ohne Druck verabreicht werden, sodass das Wasser den jeweiligen Körperteil „ummantelt“.

Der kalte Gesichtsguss ist nicht nur ganz einfach und schnell durchzuführen, er wirkt auch erstaunlich gut. Dieser Guss regt die Durchblutung im Gesicht an, strafft die Haut, wirkt erfrischend und belebend und verbessert zudem die nachlassende Sehkraft. Er wird durchgeführt, indem der Patient den Wasserstrahl am nach vorn gebeugten Kopf zunächst von der rechten Schläfe über die Stirn zur linken Schläfe und zurück führt. Anschließend wird erst die rechte, dann die linke Gesichtshälfte in Auf- und Abwärtsbewegungen und zum Schluss das Gesicht kreisförmig umrundend begossen. Wer es sich noch leichter machen möchte, kann auch zunächst die Stirn, dann die Augen und schließlich das ganze Gesicht unter die laufende Wasserleitung halten. Danach soll das Wasser vom Gesicht nur leicht abgetupft werden [3][11].

\section{Knieguss - die nichtmedikamentöse Blutdruckregulation}

Ebenfalls schnell und einfach durchführbar ist der Knieguss, der je nach Wärmehaushalt kalt oder als Wechsel- 
guss durchgeführt werden kann. Er erweitert die Arterien und wirkt daher blutdrucksenkend und durchblutungsfördernd. Er tonisiert die Venen und wirkt entstauend, vegetativ beruhigend und schlaffördernd. Zudem regt er den Knorpelstoffwechsel im Knie an und wirkt so gegen Gonarthrose [19]. Der Knieguss sollte nicht bei niedrigem Blutdruck, Menstruation, Ischialgie, Nieren- und Blasenleiden durchgeführt werden. Wie generell bei kalten Güssen sollte man vor dem Guss tief einatmen, mit Beginn des Gusses ausatmen und auf eine ruhige Atmung und entspannte Körperhaltung während des Gusses achten [5][11].

Der kalte Knieguss wird mit möglichst kaltem Wasser (max. $15^{\circ} \mathrm{C}$ ) durchgeführt und dauert nur etwa zwei Minuten. Dabei beginnt der Patient am rechten lateralen Zehenrand und führt den Wasserstrahl über den Fußrücken außen am Bein nach oben bis eine Handbreit über dem Knie, verweilt hier einen Moment und führt den Wasserstrahl dann am inneren Unterschenkel wieder abwärts bis zum Fuß. Anschließend folgt das linke Bein in gleicher Weise. Ganz zum Schluss folgen die Fußsohlen, erst die rechte, dann die linke.

Im Unterschied dazu beginnt der Wechsel-Knieguss mit $36-38^{\circ} \mathrm{C}$ warmem Wasser, das in gleicher Weise zuerst am rechten, dann am linken Bein vom Fußrücken aufwärts bis über das Knie verabreicht wird. Dieser Warmanteil wird durchgeführt, bis eine gute Durchwärmung eintritt, erst dann geht es auf der Innenseite wieder abwärts. Danach folgt der Kaltanteil wie oben beschrieben. Danach werden Warm- und Kaltanteil noch einmal wiederholt und zum Schluss beide Fußsohlen kalt abgegossen [1][5].

\section{Kalter Vollguss - Immunstimulation für Trainierte}

Die Steigerung der kleinen Güsse ist der Ganzkörper- oder Vollguss. Zusätzlich zur verbesserten Durchblutung, zur Aktivierung des Stoffwechsels und zur Straffung der Haut reguliert er den Wärmehaushalt und stimuliert das Immunsystem, das nicht nur der Infektabwehr, sondern auch der Schadstoffentsorgung dient und damit wichtige AntiAging-Aufgaben erfüllt. Wer ergänzend zu den kleinen Anwendungen regelmäßig einen Vollguss durchführt, fühlt sich nicht nur fitter, er wird auch seltener krank.

Am einfachsten bietet sich der kalte Vollguss nach einer warmen Dusche, einem warmen Vollbad oder Saunagang an. Ist der Körper gut durchwärmt, lässt sich der Kaltreiz auch gut tolerieren. Aufgrund der größeren Reizstärke ist die korrekte Durchführung dabei noch wichtiger als bei den kleinen Güssen. Wie beim Knieguss beginnt der Patient mit dem weichen, ummantelnden Wasserstrahl an der Außenseite des rechten Fußes, geht dann an der Außen- und Rückseite des Unter-, dann Oberschenkels nach oben, über das Gesäß und den Beckenkamm nach vorne zur Leiste und dann an der Innen- und Vorderseite des Beines wieder hinab zum Fuß. Danach folgt das linke
Bein in gleicher Weise. Danach kommt zuerst der rechte, dann der linke Arm, vom Handrücken über die Außenseite zur Schulter und dann innen wieder zurück. Dann entsprechend dem Gesichtsguss - folgt das Gesicht. Zum Schluss noch einmal tief einatmen und beim Ausatmen mit dem Wasserstrahl über einen Arm zu Brust und Rücken nach oben fahren und den ganzen Körper kalt abgießen. Abschließend folgen wieder die Fußsohlen, erst die rechte, dann die linke.

\section{Das Auslaugebad - Entsäuerung und „Entschlackung“}

Warme Bäder wirken vagotonisierend. Sie dilatieren die Hautgefäße, sodass die Blutmenge in der Haut auf bis zu 1,5 Liter steigt. Die Schweißsekretion steigt, was zur Eindickung des Blutes führt und über eine Sogwirkung zur Entschlackung der Körperzellen und des Zwischenzellraumes führt. Zudem wird die Darmperistaltik aktiviert.

Ein wesentlicher Faktor für die Beschleunigung von Alterungsprozessen, für Silent Inflammation und Degeneration ist die chronische Übersäuerung. Ursache hierfür sind nicht nur säurehaltige und -produzierende Nahrungsmittel, auch falsches Essverhalten, Bewegungsmangel und Stress gehören dazu. Laut Aussagen von Forschern in diesem Bereich, gibt es wohl kaum noch einen Menschen in unserer Industriegesellschaft, der nicht chronisch übersäuert ist. Somit gehören die Entsäuerung bzw. der Ausgleich des Säure-Basen-Haushaltes zu den wichtigsten Aufgaben der ganzheitlichen Anti-Aging-Strategie. Infolge ihrer ausleitenden Wirkung hat die Hydrotherapie dabei einen besonderen Stellenwert. Besonders intensiv wirken sogenannten Auslaugebäder, die sowohl als Teilbäder, vor allem als Fußbäder, als auch als Dreiviertel- oder Vollbäder durchgeführt werden können.

Im einfachsten Falle setzt sich der Patient in eine Wanne mit etwa $38^{\circ} \mathrm{C}$ warmem Wasser und seift den ganzen Körper über einen Zeitraum von etwa 20-30 Minuten intensiv mit Kernseife ein. Je wärmer und je länger das Bad durchgeführt wird, desto intensiver wirkt es. Dennoch kommt es meist nicht bei der ersten Durchführung zu einem deutlichen Ausleitungseffekt, weshalb die Prozedur möglichst an den nachfolgenden Tagen einige Male wiederholt werden sollte. Häufig bildet sich erst beim dritten bis vierten Mal ein schwer entfernbarer "Schmutzrand“ an der Badewanne, der den Ausleiteeffekt anzeigt.

Etwas eleganter und hautschonender als die Seifenmethode ist die Durchführung des Bades mit einem alkalischen Badesalz. Dieses Salz wird im Badewasser aufgelöst und der Badende verbleibt mindestens eine Stunde darin. Nachteil dieser Methode sind die höheren Kosten. Eine dritte Möglichkeit bietet Natriumbicarbonat (Natron), das ebenfalls als Badezusatz verwendet werden kann. Für ein Vollbad werden ca. $100 \mathrm{~g}$ (oder mehr) benötigt. 


\section{Sauna - Anti-Aging auf finnisch}

Wenn auch keine Hydrotherapie im Sinne Kneipps, dafür wissenschaftlich besonders gut untersucht, ist die Wirkung von Saunagängen. Diese wirken nicht nur ausleitend, abwehrsteigernd und entspannend, sie aktivieren auch die Durchblutung. Regelmäßige Saunabesuche führen zu einer deutlichen Umstimmung des vegetativen Nervensystems, senken den Sympathikotonus, stimulieren den Vagus und bewirken damit letztlich eine Senkung hoher Puls- und Blutdruckwerte [12][20]. So nimmt der mittlere funktionelle Gefäßdurchmesser im Laufe von drei Monaten um 22 \% und im Laufe von fünf Jahren um 40 \% zu [20]. Dass Saunagänge tatsächlich auch die Mortalität senken, konnte unlängst eine finnische Studie zeigen. In dieser Untersuchung wurden 2315 Männer über 20 Jahre beobachtet, die 1×, 2-3 × oder 4-7× pro Woche die Sauna besuchten. Auch die Länge der Saunagänge (weniger als 11 oder 11-19 min) wurden protokolliert. Die Forscher stellten fest, dass das Risiko für plötzlichen Herztod, Tod durch KHK oder Herz-Kreislauf-Erkrankungen, aber auch für Tod sonstiger Ursache (Gesamtmortalität) umso niedriger war, je häufiger die Person in die Sauna ging und je länger sie sich darin aufhielt [17].

\section{Die Lehmpackung - Entgiftung und Bindegewebsstärkung}

Lehm, auch Heilerde genannt, wurde bereits vor etwa fünftausend Jahren von den Ägyptern, Indern, Babyloniern und Assyrern in Form von Schlammpackungen zu Heilzwecken und in der Kosmetik verwendet. Zu Beginn des 20. Jahrhunderts war es Pastor Felke, der viele Heilungssuchende in Deutschland damit behandelte: Tägliche Lehmbäder in für jeden Patienten eigens ausgehobenen Gruben machten die Patienten wieder gesund oder führten zumindest zu einer nachhaltigen Linderung ihrer Beschwerden [10].
Lehm besteht aus feinem Gesteinsstaub, der durch Verwitterung entstanden ist. Er enthält viele Mineralien und Spurenelemente wie Kalzium, Kalium, Magnesium und Eisen und ist besonders reich an Kieselsäure, die den Hauptbestandteil unseres Bindegewebes ausmacht. Die Spannkraft und Elastizität unseres Körpers hängen wesentlich vom Gehalt an Kieselsäure ab. Der Mangel daran führt zu vorzeitiger Alterung, Faltenbildung und Bindegewebsschwäche. Unabhängig von seiner mineralischen und damit chemischen Zusammensetzung hat Lehm eine große spezifische Oberfläche von ca. $6 \mathrm{~m}^{2} / \mathrm{g}$, die diesen Wirkstoff als sehr wirksames Adsorbens für gelöste und gasförmige Stoffe prädestiniert. Damit wirkt er gleich zweifach: Einerseits entzieht er dem Körper Krankheitsgifte, die zu Entzündung, Schwellung und Schmerzen im Gewebe führen, andererseits gibt er wichtige Mineralien und Spurenelemente, vor allem Kieselsäure, an den Organismus ab, wodurch es zur schnelleren Wundheilung, Kräftigung des Bindegewebes und Regeneration kommt. Da Lehm wasserundurchlässig ist und der für die Behandlung genutzte Lehm aus mehreren Metern Tiefe gewonnen wird, ist er praktisch keimfrei und enthält keinerlei Umweltgifte.

Aufgrund dieser Eigenschaften bieten Lehmpackungen eine ideale Möglichkeit zur Straffung und Durchblutungsverbesserung der Haut und Entschlackung des Bindegewebes im Gesicht und an Problemzonen [18]. Ein weiteres wichtiges Anwendungsgebiet sind die Gelenke. Degenerative Erkrankungen des Bewegungsapparates sind typische Alterserscheinungen, die sich durch Lehmpackungen - ggf. im Wechsel mit Güssen - deutlich verbessern oder gar vermeiden lassen. So konnten italienische und türkische Wissenschaftler nachweisen, dass Lehm- bzw. Schlammpackungen die Aktivität der Knorpelzellen (Chondrozyten) bei Arthrosepatienten durch die Modulation der Zytokinproduktion im Serum (wie Interleukin 1) beeinflussen und antientzündlich wirken [2][13]. Auch die Schmerz- 
linderung und Steigerung der Lebensqualität konnte belegt werden [6][8].

Dazu wird der trockene Lehm in kaltem Wasser angerührt und etwa messerrückendick auf die Haut aufgetragen. Anschließend werden das eingepackte Gebiet in Tücher und der Patient in mehrere Decken fest eingepackt, wo er dann 1-2 Std ruht, bis der Lehm gut durchgetrocknet ist. Danach wird der trockene Lehm abgespült und eine Nachruhe von 0,5-1 Std eingehalten. Ganz einfach anzuwenden ist eine Gesichtsmaske aus Lehm oder Heilerde, die nicht abgedeckt werden muss. Lehmauflagen und -wickel eignen sich besonders zur kalten Anwendung. Für kälteempfindliche Patienten besteht die Möglichkeit, eine Wärmflasche an die Füße zu legen, da nur der gut durchwärmte Patient richtig vom Lehm profitiert [8].

\section{Interessenkonflikt}

Der Autor erklärt, dass keine wirtschaftlichen oder persönlichen Verbindungen bestehen.

Autor

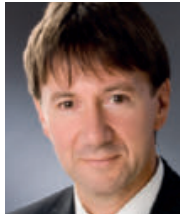

\section{Peter W. Gündling}

Arzt für Allgemeinmedizin, Naturheilverfahren, Akupunktur, Homöopathie, Chirotherapie, Sportmedizin, Ernährungsmediziner, Badearzt, seit 1988 in eigener Praxis niedergelassen. Seit 2006 Professor für Naturheilkunde und komplementäre Medizin an der Hochschule Fresenius in Idstein, wo er als Studiendekan ein neues Masterstudium zur Naturheilkunde und komplementären Medizin für Ärzte entwickelt hat und leitet. Studiendekan für Naturheilkunde und komplementäre Medizin, Carl Remigius Medical School. Lehrbeauftragter der Johann-Wolfgang-Goethe-Universität Frankfurt a. Main

\section{Korrespondenzadresse}

Prof. Dr. med. Peter W. Gündling MME M.Sc.

Sebastian-Kneipp-Str. 4

65520 Bad Camberg/Ts.

E-Mail: guendling@hs-fresenius.de
[4] Brenke R, Conradi E. Hydrotherapie. In: Kraft K, Stange R, Hrsg. Lehrbuch Naturheilverfahren. Stuttgart: Hippokrates; 2010: 181-208

[5] Brüggemann W. Kneipp-Therapie. Ein Lehrbuch. Berlin: Springer; 1980

[6] Espejo Antúnez L, Caro Puértolas B, Ibáñez Burgos B et al. Effects of mud therapy on perceived pain and quality of life related to health in patients with knee osteoarthritis. Reumatol Clin 2013; 9(3): 156-60. doi: 10.1016/j. reuma.2012.09.005

[7] Fiscus KA, Kaminski TW, Powers ME. Changes in lower-leg blood flow during warm-, cold-, and contrast-water therapy. Arch Phys Med Rehabil 2005; 86(7): 1404-1410

[8] Guendling PW, Schaedle S, Jork K. Therapeutic Effects of mud packs on joint ailment and quality of life from patients with gonarthrosis - a controlled clinical study. Europ J of Integrative Med 2009; 1(4): 242

[9] Gündling PW. Brennpunkt Herz. Natürlich heilen und vorbeugen bei Herz-Kreislauf-Problemen. Baden-Baden: Aurelia; 2004

[10] Gündling, PW. Lehmpackungen und Wasseranwendungen bei Venenleiden. Praxis Magazin 2012; 29(2): 6-11

[11] Gündling PW. Wasser kontra Schmerzen - Stellenwert der Hydrotherapie in der Schmerzbehandlung. Erfahrungsheilkunde 2018; 67(3): 148-153

[12] Gündling PW. Blutdruckregulation durch Wasserreize. Hydrotherapie nach Kneipp bei art. Hypertonie. Erfahrungsheilkunde 2014; 63(1): 5-13. Im Internet: http://dx.doi. org//10.1055/s-0033-1357608; Stand: 30.01.2019

[13] Güngen G, Ardic F, Fındıkoğlu G et al. The effect of mud pack therapy on serum YKL-40 and hsCRP levels in patients with knee osteoarthritis. Rheumatol Int 2012; 32(5): 1235-44. doi: $10.1007 /$ s00296-010-1727-4

[14] Hahn T. Wasser thut's freilich. 1776

[15] Kneipp S. Meine Wasser-Kur. 50. Aufl. Kempten: Kösel; 1888.

[16] Kneipp S. So sollt ihr leben. 1. Aufl. Kempten: Kösel; 1889

[17] Laukkanen T, Khan H, Zaccardi F, Laukkanen JA. Association between sauna bathing and fatal cardiovascular and all-cause mortality events. JAMA Intern Med 2015; 175(4): 542-8

[18] Poensin D, Carpentier PH, Féchoz C et al. Effects of mud pack treatment on skin microcirculation. Joint Bone Spine 2003; 70: $367-370$

[19] Schencking M, Wilm S, Redaelli M. A comparison of Kneipp hydrotherapy with conventional physiotherapy in the treatment of osteoarthritis: a pilot trial. J Integr Med 2013 Jan; 11(1): 17-25. doi: 10.3736/jintegrmed2013004

[20] Winterfeld H], Siewert H, Strangfeld D. Der Einsatz der Sauna zur Therapie und Prophylaxe der essentiellen Hypertonie im Stadium I und II unter besonderer Berücksichtigung der peripheren und zentralen Hämodynamik. Int Sauna-Arch 1986; 3: 37-46

\section{Bibliografie}

DOI https://doi.org/10.1055/a-0836-7628

EHK 2019; 68: 22-28

(c) MVS Medizinverlage Stuttgart GmbH \& Co. KC ISSN 0014-0082 